\title{
Mixed Profile Method of Speed and Location for Robotic Arms Motion used for Precise Positioning
}

\author{
Liliana Marilena Matica ${ }^{1}$, Cornelia Győrödi' ${ }^{2}$, \\ Helga Silaghi ${ }^{3}$ \\ Faculty of Electrical Engineering and Information \\ Technology, University of Oradea \\ Oradea, Romania
}

\author{
Andrei Silaghi ${ }^{4}$ \\ Faculty of Electronics and Telecommunications \\ Engineering, University Politehnica Timişoara, \\ Timişoara, Romania
}

\begin{abstract}
The paper describes a new real-time computation method named Mixt Profile of Speed (MPS), which is used to obtain the value of speed, at every sampling period of time, during the acceleration and deceleration stage, whereas the motion has three stages: 1) acceleration, 2) motion with imposed constant speed, and 3) deceleration. The method will determinate the location of a robotic arm for every sampling period of time. The originality of this new computation method refers to the deceleration stage; it determines an accurate positioning at the end of the motion in a well determinate interval of time. During the forced constant motion stage, the trajectory is imposed and it is linear or circular. The ADNIA algorithm (numerical differential analysis interpolation algorithm) can be implemented at this stage (during the motion with imposed constant speed of the robotic arm) in order to ensure the maximum precision of the computation for the waypoints Cartesian coordinates.
\end{abstract}

Keywords-Sampling period of time; waypoints; location matrix for a robotic arm; acceleration; deceleration; motion stage; mixt profile of speed; trapezoidal profile; parabolic profile

\section{INTRODUCTION}

The robotic technology is used in various industry sectors [1], [2], [6], [7]. The problems that arise are complexity of planning a robot motion and real-time computation of the speed and location for the robotic arm motion; these can be computationally intensive and time-consuming. One approach described in textbooks [4], [5] generates a trajectory that satisfies acceleration and speed constraints from a list of waypoints and use linear segments with parabolic blends. The approach is not applicable to automatically generated paths with potentially dense waypoints. In [2] was presented a method to generate the time optimal trajectory along a given path within given bounds on acceleration and speed. The method assumes that the acceleration and speed of individual coordinates are limited. In [3] was proposed to reduce the computation time for path planning of motion for a robotic arm by use to the techniques of caching frequent arm trajectories. In Fig. 1 is presented the block diagram of the robotic system.

The challenges of motion for robotic arm, involve finding the best precision for reaching the end point of motion, also it is important to obtain precise value about required motion time. Both conditions are very difficult to be obtained. The proposed real-time computation method in this paper, namely, Mixt Profile of Speed (named shortly MPS), defined and described in this paper, accomplished those two conditions, [10].

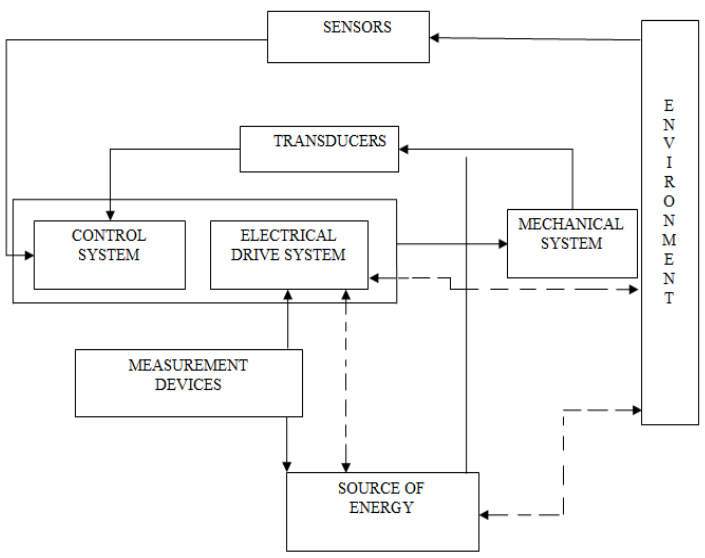

Fig. 1. Block diagram of the robotic system.

Usually, regarding robotic arm motion [1]-[3], the speed variation profile may have a trapezoidal profile, as in Fig. 2:

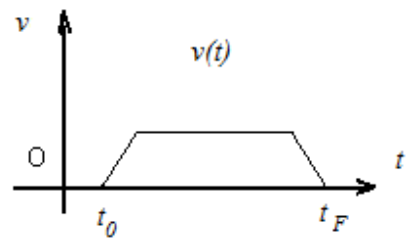

Fig. 2. Trapezoidal profile of speed.

or a parabolic profile, as presented in Fig. 3:

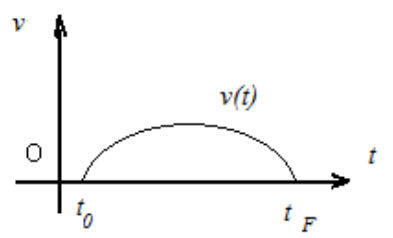

Fig. 3. Parabolic profile of speed.

In previous figures, index 0 shows the start of motion and index $F$ shows the motion stop.

This paper describes another profile of motion speed, named MPS (Mixt Profile of Speed), as in Fig. 4: 


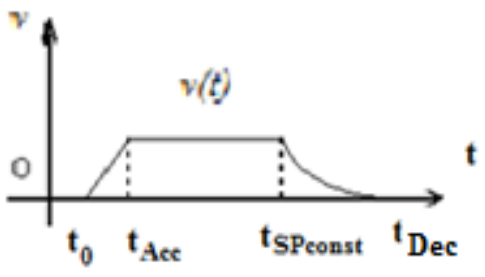

Fig. 4. Mixt profile of speed.

In previous figure, index Acc defines the end of acceleration process, index SPconst defines the end of motion with constant imposed speed and index Dec defines the end of deceleration process.

The motion with a mixt profile of speed supposes three motion stages:

a) acceleration stage (the first stage of motion);

b) motion with constant imposed speed (the second stage of motion);

c) deceleration stage (the third stage of motion).

The MPS profile of speed variation ensures a precise positioning, at the end of the motion. It may be implementing about motion with a well-defined constant speed, on a linear or circular imposed trajectory [9].

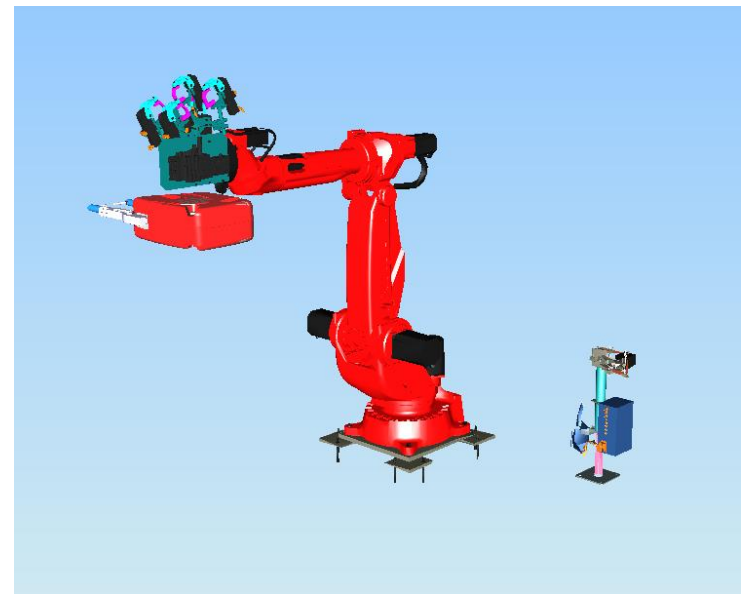

Fig. 5. Industrial robotic arm.

About a robotic arm as shown in Fig. 5, the motion speed value determines the axle components, named: $\vec{p}_{x} ; \vec{p}_{y} ; \vec{p}_{z}$ of position vector (named: $\vec{p}$ presented in Fig. 5), in the location matrix of a robotic arm [8]:

$$
G=\left[\begin{array}{cccc}
n_{x} & o_{x} & a_{x} & p_{x} \\
n_{y} & o_{y} & a_{y} & p_{y} \\
n_{z} & o_{z} & a_{z} & p_{z} \\
0 & 0 & 0 & 1
\end{array}\right]
$$

About the location matrix, the others values are the axle components of the orientation versors: $\vec{n} ; \vec{o} ; \vec{a}$ (a versor is a vector having module equal with 1 , because its size is not important; only its orientation is important), Fig. 6 [1]-[3].
This real-time computation method was implemented about positioning pieces, in a flexible manufacturing cell for welding industrial process. The computation method has not been mentioned about robotic arms motion; this paper adapts the computation method for robotic arms motion.

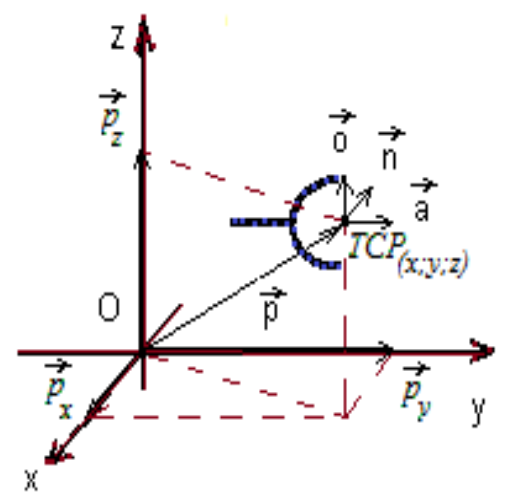

Fig. 6. Position vector for a robotic arm; orientation versors.

The next section presents a new real-time computation method named Mixt Profile of Speed (MPS), which is used to obtain the value of speed, at every sampling period of time, during the acceleration and deceleration stage. The motion with a mixt profile of speed has three stages: acceleration stage, motion with constant imposed speed and deceleration stage. Based on the results of the tests performed, several conclusions are presented in the last section.

\section{ACCELERATION Stage, Stage of Motion With CONSTANT IMPOSED SPEED AND DECELERATION STAGE OF MOTION}

The real-time computation method named mixt profile of speed (MPS) is described in this paragraph [1].

\section{A. Acceleration Stage}

Let consider the maximum value of acceleration for a sampling period of time, named: $a c c_{\text {Max }}$; the initial value of speed at the motion start: $v_{0}$. At every sampling period of time, named $\mathrm{T}$, the speed increases with $\operatorname{acc}_{\operatorname{Max}}$ value, till reach the imposed value, named: $v_{-}$impoz. It result the value of motion speed, at every sampling period of time, indexed $m$, (during the acceleration stage):

$$
v_{m}=v_{m-1}+T \cdot a c c_{\operatorname{Max}}=v_{0}+m \cdot T \cdot a c c_{\operatorname{Max}}
$$

This computation must be considered for every axle component of speed:

$$
\begin{aligned}
& v_{x, m}=v_{x, m-1}+T \cdot a c c_{\text {Max }}=v_{x, 0}+m \cdot T \cdot a c c_{\text {Max }} \\
& v_{y, m}=v_{y, m-1}+T \cdot a c c_{\text {Max }}=v_{y, 0}+m \cdot T \cdot a c c_{\text {Max }} \\
& v_{z, m}=v_{z, m-1}+T \cdot a c c_{\text {Max }}=v_{z, 0}+m \cdot T \cdot a c c_{\text {Max }}
\end{aligned}
$$


It result the variation of position vector, for every sampling period of time (named $T$ ), during acceleration stage of motion:

$$
\begin{aligned}
& \vec{p}_{x, m}=\vec{p}_{x, m-1}+T \cdot v_{x, m} \cdot \vec{i} \\
& \vec{p}_{y, m}=\vec{p}_{y, m-1}+T \cdot v_{y, m} \cdot \vec{j} \\
& \vec{p}_{z, m}=\vec{p}_{z, m-1}+T \cdot v_{z, m} \cdot \vec{k}
\end{aligned}
$$

For example, let consider this example of computation: the location matrix of the robotic arm, at the motion start, index 0 (the values are expressed in millimetres, $[\mathrm{mm}]$ ) is:

$$
G_{0}=\left[\begin{array}{cccc}
n_{x, 0} & o_{x, 0} & a_{x, 0} & p_{x, 0} \\
n_{y, 0} & o_{y, 0} & a_{y, 0} & p_{y, 0} \\
n_{z, 0} & o_{z, 0} & a_{z, 0} & p_{z, 0} \\
0 & 0 & 0 & 1
\end{array}\right]=\left[\begin{array}{cccc}
1 & 0 & 0 & 3 \\
0 & 1 & 0 & 5 \\
0 & 0 & 1 & 7 \\
0 & 0 & 0 & 1
\end{array}\right]
$$

Let consider the value of sampling period of time $T=0.01$ seconds [s]. Let consider the initial speed having 0 value (the start motion value of speed) and the maximum value of acceleration: $a c c_{\text {Max }}=200[\mathrm{~mm} / \mathrm{s}]$ (identical for every axle); about the first three sampling periods of time, the speed has those OX axle components values:

$$
\begin{aligned}
& v_{x, 1}=v_{x, 0}+1 \cdot T \cdot a c c_{\text {Max }}=0+1 \cdot 0.01 \cdot 200=2=v_{y, 1}=v_{z, 1} \\
& v_{x, 2}=v_{x, 0}+2 \cdot T \cdot a c c_{\text {Max }}=0+2 \cdot 0.01 \cdot 200= \\
& =4=v_{y, 2}=v_{z, 2} \\
& v_{x, 3}=v_{x, 0}+3 \cdot T \cdot a c c_{\text {Max }}=0+3 \cdot 0.01 \cdot 200=6=v_{y, 3}=v_{z, 3}
\end{aligned}
$$

The OX axle components of position vector, for the first three sampling period of time, during acceleration stage of motion have those values:

$$
\begin{aligned}
& \vec{p}_{x, 1}=\vec{p}_{x, 0}+T \cdot v_{x, 1} \cdot \vec{i}=(3+0.01 \cdot 2) \cdot \vec{i}=3.02 \cdot \vec{i} \\
& \vec{p}_{x, 2}=\vec{p}_{x, 1}+T \cdot v_{x, 2} \cdot \vec{i}=(3.02+0.01 \cdot 4) \cdot \vec{i}=3.06 \cdot \vec{i} \\
& \vec{p}_{x, 3}=\vec{p}_{x, 2}+T \cdot v_{x, 3} \cdot \vec{i}=(3.06+0.01 \cdot 6) \cdot \vec{i}=3.12 \cdot \vec{i}
\end{aligned}
$$

In previous relations (rel.7), versor $\vec{i}$ is the OX axle versor (it has the module equal with $l$ value and the orientation along the positive sense of this axle); also, in this paper, versor $\vec{j}$ is the $\mathrm{OY}$ axle versor and versor $\vec{k}$ is the $\mathrm{OZ}$ axle versor.

Similar computation (as rel.7) must be implemented about $\mathrm{OY}$ and $\mathrm{OZ}$ axle components of position vector; it results next values: $\vec{p}_{y}=5.12 \cdot \vec{j}$ and $\vec{p}_{z}=7.12 \cdot \vec{k}$; so, after 3 periods of time, the location matrix of the robotic arm is:

$$
G_{3}=\left[\begin{array}{cccc}
1 & 0 & 0 & p_{x, 3} \\
0 & 1 & 0 & p_{y, 3} \\
0 & 0 & 1 & p_{z, 3} \\
0 & 0 & 0 & 1
\end{array}\right]=\left[\begin{array}{cccc}
1 & 0 & 0 & 3.12 \\
0 & 1 & 0 & 5.12 \\
0 & 0 & 1 & 7.12 \\
0 & 0 & 0 & 1
\end{array}\right]
$$

In the acceleration stage, about the last sampling period of time, the considered acceleration may be less then maximum value of acceleration, in order to reach the imposed constant speed. Let consider the number of sampling period of time required for acceleration stage, named: $N_{A c c}$; it results the acceleration for the last sampling period of time:

$$
a c c_{N_{A c c}}=v_{\text {impoz }}-\left(N_{A c c}-1\right) \cdot a c c_{\text {Max }}
$$

For example, if the maximum acceleration value is 200 $\mathrm{mm} / \mathrm{s}$; the value of sampling period of time $T=0.01 \mathrm{~s}$. The speed at the motion start has $O$ value and the constant imposed speed is $9 \mathrm{~mm} / \mathrm{s}$, it results the speed values, during the acceleration stage, at every sampling period of time, having those values: $0 ; 2 ; 4 ; 6 ; 8 ; 9 \mathrm{~mm} / \mathrm{s}$ (for the last period of time, during acceleration stage.

The acceleration has the value of $100 \mathrm{~mm} / \mathrm{s}$, which is the maximum value possible). It results the number of sampling period of time for acceleration stage (named: $N_{A c c}$ ), about this example is 5 .

\section{B. Motion with Imposed Constant Speed}

The next motion stage is performed with the constant imposed value of speed, named: $v_{\text {impoz }}$. Usually, motion upon an imposed well-defined trajectory (linear or circular) is executed with an imposed constant speed.

Let consider an imposed linear trajectory and the axle components of the imposed constant speed: $v_{X, \text { impoz }} ; v_{Y, \text { impoz }}$; $v_{Z, \text { impoz }}$. It results the axle steps, executed by the robotic arm, named: $\delta_{x} ; \delta_{y} ; \delta_{z}$, at every sampling period of time (named $T)$,during this motion stage:

$$
\begin{aligned}
& \delta_{x}=T \cdot v_{X, \text { impoz }} \\
& \delta_{y}=T \cdot v_{Y, \text { impoz }} \\
& \delta_{z}=T \cdot v_{Z, \text { impoz }}
\end{aligned}
$$

It results the axle components of position vector, indexed $l$, (index $l$ starts with 1 value), for every sampling period of time, during this motion stage:

$$
\begin{aligned}
& \vec{p}_{x, l}=\vec{p}_{x, l-1}+\delta_{x} \cdot \vec{i}=\vec{p}_{x, N_{A c c}}+l \cdot \delta_{x} \cdot \vec{i} \\
& \vec{p}_{y, l}=\vec{p}_{y, l-1}+\delta_{y} \cdot \vec{j}=\vec{p}_{y, N_{A c c}}+l \cdot \delta_{y} \cdot \vec{j}
\end{aligned}
$$




$$
\vec{p}_{z, m}=\vec{p}_{z, m-1}+\delta_{z} \cdot \vec{k}=\vec{p}_{z, N_{A c c}}+l \cdot \delta_{z} \cdot \vec{k}
$$

In previous relation (rel.11), $\vec{p}_{x, N_{A c c}} ; \vec{p}_{y, N_{A c c}} ; \vec{p}_{z, N_{A c c}}$ are the initial values of axle components of position vector, about the second stage of motion; those values are identical with position vector axle component values, at the end of the first stage of motion, the acceleration stage.

For example, let considers those values: $\vec{p}_{x, N_{A c c}}=10 \cdot \vec{i}$ $[\mathrm{mm}] ; \quad \vec{p}_{y, N_{A c c}}=100 \cdot \vec{j}[\mathrm{~mm}] ; \quad \vec{p}_{z, N_{A c c}}=1000 \cdot \vec{k} \quad[\mathrm{~mm}]$. Let considers the axle steps having those values of millimetres: $\delta_{x}=1.11[\mathrm{~mm}] ; \delta_{y}=1.22[\mathrm{~mm}] ; \delta_{z}=1.33[\mathrm{~mm}]$. It results the axle components of position vector, at every sampling period of time (index $l$ ), during second motion stage:

$$
\begin{aligned}
& \vec{p}_{x, l}=\vec{p}_{x, N_{A c c}}+l \cdot \delta_{x} \cdot \vec{i}=10 \cdot \vec{i}+l \cdot 1.11 \cdot \vec{i} \\
& \vec{p}_{y, l}=\vec{p}_{x, N_{A c c}}+l \cdot \delta_{y} \cdot \vec{j}=100 \cdot \vec{j}+l \cdot 1.22 \cdot \vec{j} \\
& \vec{p}_{z, l}=\vec{p}_{x, N_{A c c}}+l \cdot \delta_{z} \cdot \vec{k}=1000 \cdot \vec{k}+l \cdot 1.33 \cdot \vec{k}
\end{aligned}
$$

Considering rel.12, it may be computed the position vector, at every sampling period of time, during the motion with constant imposed speed (the second stage of motion). For example, after 200 sampling period of time, the axle component of position vector have the values:

$$
\begin{aligned}
& \vec{p}_{x, 200}=10 \cdot \vec{i}+200 \cdot 1.11 \cdot \vec{i}=(10+222) \cdot \vec{i}=121 \cdot \vec{i} \\
& \vec{p}_{y, 200}=100 \cdot \vec{j}+200 \cdot 1.22 \cdot \vec{j}=344 \cdot \vec{j} \\
& \vec{p}_{z, 200}=1000 \cdot \vec{k}+200 \cdot 1.33 \cdot \vec{k}=1266 \cdot \vec{k}
\end{aligned}
$$

It result the location matrix for every sampling period of time. In order to work with different index value about location matrix during all three motion stages, it must be considered: $N_{\text {Acc }}$; so, during motion with constant speed, (index $l$ ), the location matrix is:

$$
G_{l+N_{A c c}}=\left[\begin{array}{cccc}
1 & 0 & 0 & p_{x, l} \\
0 & 1 & 0 & p_{y, l} \\
0 & 0 & 1 & p_{z, l} \\
0 & 0 & 0 & 1
\end{array}\right]=\left[\begin{array}{cccc}
1 & 0 & 0 & 121 \\
0 & 1 & 0 & 344 \\
0 & 0 & 1 & 1266 \\
0 & 0 & 0 & 1
\end{array}\right]
$$

In previous relations (rel.11; rel12; rel.14), the index $l$ goes from 1 (one) value to $N_{S P c o n s t}$. The value of $N_{\text {SPconst }}$ results from conditions defined about others two motion stage (as it follows the explanations). So, the distance performed during the acceleration stage (the first stage of motion) and deceleration stage (the third and the last stage of motion) must be computed.

The start point and the stop point, about motion upon the imposed linear trajectory, must be defined; it results the entire distance of motion. Deducting from entire distance of motion, it result the distance of the second motion stage (motion with constant imposed speed). Considering this distance value and the value of imposed constant speed, it results the time required for the second motion stage. It must be divided this time value to the value of sampling period of time; so, it results $N_{S P c o n s t}$ (last index value about second stage of motion).

During first and second motion stage, the required number of sampling period of time is: $N_{A c c}+N_{S P c o n s t}$.

\section{Deceleration Stage of Motion}

About the third stage, the deceleration stage of motion (the last motion stage), the variation of speed (indexed $q$ ) is:

$$
v_{q}=v_{q-1}-T \cdot d e c_{q}
$$

The deceleration value, named $d e c_{q}$, has not a constant value, it has a decreasing value, in purpose to ensure a precise positioning, at the motion end (the $b$ value is a constant and adjusts the decreasing of speed with several others characteristics of motion):

$$
\operatorname{dec}_{q}=\frac{b}{q^{2}}
$$

For example, let consider $b=50$, it results the values of deceleration: $\operatorname{dec}_{1}=50 \mathrm{~mm} / \mathrm{s}^{2} ; \quad \operatorname{dec}_{2}=12.5 \mathrm{~mm} / \mathrm{s}^{2} ; \quad \operatorname{dec}_{3}=5.55$ $\mathrm{mm} / \mathrm{s}^{2} ; \operatorname{dec}_{4}=3.11 \mathrm{~mm} / \mathrm{s}^{2} ; \operatorname{dec}_{5}=2 \mathrm{~mm} / \mathrm{s}^{2}$.

During the deceleration stage, the speed decreases, till it reaches the 0 value.

The time lapse, during the deceleration stage, is very well defined, linked with the number of sampling period of time required for the deceleration stage, named $N_{D e c}$ (index $q$ goes from 1 value to $N_{D e c}$ value). It results from those conditions: it begin with $v_{\text {impoz }}$ and end with $O$ value of speed, about deceleration stage:

$$
\begin{aligned}
& v_{\text {impoz }}=v_{q=0} \\
& v_{N_{\text {Dec }}}=0
\end{aligned}
$$

The computation method must consider the different values of speed axle components; so, the previous conditions must be applied for the maximum value of speed axle component:

$$
\mathrm{v}_{\mathrm{q}=1}=\max \left(\mathrm{v}_{\mathrm{x}, \text { impoz }} ; \mathrm{v}_{\mathrm{y}, \text { impoz }} ; \mathrm{v}_{\mathrm{z}, \text { impoz }}\right)
$$

Considering the previous computation example, this value is the maximum from: $111 \mathrm{~mm} / \mathrm{s} ; 122 \mathrm{~mm} / \mathrm{s}$ and $133 \mathrm{~mm} / \mathrm{s}$.

The number (named $N_{D e c}$ ) of sampling period of time, required for deceleration stage, is:

$$
\max \left(v_{x, \text { impoz }} ; v_{y, \text { impoz }} ; v_{z, \text { impoz }}\right)=\frac{b}{\left(N_{\text {Dec }}\right)^{2}}
$$


The resulting number must be the next integer value, greater then: $\sqrt{\frac{b}{v_{\text {impoz }}}}$ because the end point approach must be done with a very small deceleration.

Considering the previous example of deceleration for OX axle component of speed, the computation of this axle component starts with those relations:

$$
\begin{aligned}
& v_{x, 2}=v_{x, \text { impoz }}-T \cdot d e c_{x, 1}=v_{x, \text { impoz }}-T \cdot 50 \mathrm{~mm} / \mathrm{s}^{2} \\
& v_{x, 3}=v_{x, 2}-T \cdot d e c_{x, 2}=v_{x, 2}-T \cdot 12.5 \mathrm{~mm} / \mathrm{s} \\
& v_{x, 4}=v_{x, 3}-T \cdot d e c_{x, 3}=v_{x, 3}-T \cdot 5.55 \mathrm{~mm} / \mathrm{s}
\end{aligned}
$$

The initial value of speed for deceleration stage (index $q=0$ ) is the imposed constant speed named: $v_{\text {impoz }}$ (for motion on the imposed trajectory).

The similar computation, about OX axle component of speed, must be applied about $\mathrm{OY}$ and $\mathrm{OZ}$ axle component of speed, so, the computation of axle components of speed is:

$$
\begin{aligned}
& v_{x, q}=v_{x, q-1}-T \cdot d e c_{x, q} \\
& v_{y, q}=v_{y, q-1}-T \cdot d e c_{y, q} \\
& v_{z, q}=v_{z, q-1}-T \cdot d e c_{z, q}
\end{aligned}
$$

Index $q$ goes from 1 value to $N_{D e c}$ value, (computed with rel.21). For each speed axle component, the last value of deceleration must be adjusted to the proper value, in order to obtain 0 value of speed.

It results the computation of position vector (index $q$ ):

$$
\begin{aligned}
& \vec{p}_{x, q}=\vec{p}_{x, q-1}+T \cdot v_{x, q} \cdot \vec{i} \\
& \vec{p}_{y, q}=\vec{p}_{y, q-1}+T \cdot v_{y, q} \cdot \vec{j} \\
& \vec{p}_{z, q}=\vec{p}_{z, q-1}+T \cdot v_{z, q} \cdot \vec{k}
\end{aligned}
$$

The forth column of location matrix for robotic arm contains those axle components of position vector (as it was explained previously), the location matrix during deceleration stage is:

$$
G_{q+N_{\text {Acc }}+N_{\text {SPconst }}}=\left[\begin{array}{cccc}
1 & 0 & 0 & p_{x, q} \\
0 & 1 & 0 & p_{y, q} \\
0 & 0 & 1 & p_{z, q} \\
0 & 0 & 0 & 1
\end{array}\right]
$$

The motion with mixt profile of speed needs $N_{\text {Acc }}+N_{\text {SPconst }}+N_{\text {Dec }}$ sampling periods of time (as it was explained).

\section{CONCLUSIONS}

The paper defines and describes the real-time computation method named mixt profile of speed (speed variation), for motion on an imposed linear trajectory.

The motion implementing mixt profile of speed has three stages: acceleration stage; motion with constant imposed speed; deceleration stage.

The paper shows the real-time computation of mixt profile of speed, for a robotic arm motion, on a linear trajectory. For each of the three stages of motion, the position vector may be computed with relations (4), (12) and (23), thus it results the location matrix of the robotic arm relations (5), (14) and (24).

The proposed MPS method implements the maximum computation precision, for robotic arm motion, upon an imposed linear trajectory, with a constant imposed speed.

The method offers the best precision for reaching the end point of motion; also it obtains a precise value for required motion time.

\section{REFERENCES}

[1] L.M.Matica, H. Oros, "Speed Computation for Industrial Robots Motion Followed by Accurate Positioning. International Journal of Computers", Communications \& Control, ISSN 1841-9836, 12(1):76-89, February 2017.

[2] T. Kunz, M. Stilman, "Time-Optimal Trajectory Generation for Path with Bounded Acceleration and Velocity", Robotics: Science and Systems $8^{\text {th }}$ Conference, July 9-13, 2012, Sydney, Australia, ISBN 978-0-262-51968-7, pp. 209-216.

[3] K. Krishnaswamy, J. Sleeman, T. Oates, "Real-Time Path Planning for Robotic Arm", Proceedings of the 4th International Conference on Pervasive Technologies Related to Assistive Environments , May 25-27, 2011, ISBN: 978-1-4503-0772-7, doi:10.1145/2141622.2141635.

[4] J.J. Craig, "Introduction to Robotics: Mechanics and Control $\left(3^{\text {rd }}\right.$ Edition)", Prentice Hall, 2004, ISBN-10: 0133489795.

[5] B. Siciliano, L. Sciavicco, L. Villani, G. Oriolo, "Robotics: moddelling, planning and control", Springer 2009, ISBN 978-1-84628-641-4.

[6] Z. Shiller, H.H. Lu. "Computation of path constrained time optimal motions with dynamic singularities", Journal of Dynamic Systems, Measurement, and Control, Vol 114, pp. 34 - 40, 1992.

[7] M. Zucker, N. Ratliff, A.D. Dragan, M. Pivtoraiko, M. Klingensmith, C. M. Dellin, J.A. Bagnell, S.S. Srinivasa, "CHOMP, Covariant hamiltonian optimization for motion planning “, Int. Journal of Robotics Research, 2012.

[8] L.M.Matica, C.Gyorodi, H.Silaghi, S.Abrudan Caciora, "Real Time Computation for Robotic Arm Motion upon a Linear or Circular Trajectory“, International Journal of Advanced Computer Science and Applications, Vol.9, Issue 2, 2018.

[9] S.Dale, Helga Silaghi, D. Zmaranda, U. Rohde, "Intelligent Design Environment for Second-Order Positioning Systems", International Journal of Computers Communications and Control, ISSN 1841-9836, Vol.10, No 1, 2015.

[10] C.R. Costea, Helga Silaghi, D. Zmaranda, M.A. Silaghi, "Control Systems Architecture for a Cement Mill based on Fuzzy Logic“, International Journal of Computers Communications and Control, ISSN 1841-9836, Vol.10, No 2, 2015. 